\title{
A Novel Threshold Scheme for Reducing Spatial Intersymbol Interference in Vehicular Visible Light Communication Based on Image Sensors
}

\author{
Ling Zhan $\mathbb{D}^{1,2}$ and Hong Zhao $\mathbb{D}^{2}$ \\ ${ }^{1}$ Guangxi Key Laboratory of Wireless Broadband Communication and Signal Processing, Guilin University of \\ Electronic Technology, 541004 Guilin, China \\ ${ }^{2}$ School of Information and Communication, Guilin University of Electronic Technology, 541004 Guilin, China \\ Correspondence should be addressed to Hong Zhao; zhaohong@guet.edu.cn
}

Received 14 June 2021; Revised 11 August 2021; Accepted 28 August 2021; Published 22 September 2021

Academic Editor: Renchao Xie

Copyright (C) 2021 Ling Zhan and Hong Zhao. This is an open access article distributed under the Creative Commons Attribution License, which permits unrestricted use, distribution, and reproduction in any medium, provided the original work is properly cited.

\begin{abstract}
LEDs can be used to communicate under the premise of satisfying illumination, which lays the groundwork for visible light communication (VLC). The application of outdoor VLC has drawn more attention, especially the application of vehicle-to-vehicle $(\mathrm{V} 2 \mathrm{~V})$ which can significantly improve traffic safety and information transmission efficiency. The image sensor has the characteristics of spatial separation, easily realizes MIMO, and does not need to be strictly aligned with the transmitter, so it is especially suitable for vehicular VLC. However, because of the diffuseness of LED and the small pixel size of the image sensor, it is easy to produce the blooming effects and interfering among symbols. When decoding at the receiving terminal, it will judge the status Off of LEDs to be the status On. By analysing the blooming effects caused by surrounding LEDs, a novel threshold scheme is proposed in this paper according to the probability distribution of the sum of the optical power of the LED itself and after interference. Experiments show that the proposed method has advantages in improving BER compared with the expected threshold method and the average method, especially with long-distance communication $(70 \mathrm{~m})$, and the BER has been improved by $57 \%$ and $39 \%$, respectively.
\end{abstract}

\section{Introduction}

With the continuous decline of price and the improvement of lighting efficiency, light-emitting diode (LED) is gradually replacing the traditional light source with its long service life, energy saving, and low price [1-4]. In recent years, due to the lack of radio frequency $(\mathrm{RF})$ bandwidth in traditional wireless communication, visible light communication (VLC) as a supplement to the future sixth-generation (6G) wireless communication has been drawing people's attention [5-7]. In VLC, the transmitter can send information through the high-speed flashing of LED. After the channel transmission, the receiver uses photodiodes (PDs) or image sensors (IS) to receive the signal. At the present stage of vehicles, light sources such as headlights, taillights, and brake lights have been installed, which can increase the illumination in the daytime. These fixed light sources are mostly composed of LEDs. Cameras based on image sensors used as receivers also appear on most vehicles in the form of the dashcam, making it easy to realize vehicle to vehicle $(\mathrm{V} 2 \mathrm{~V})$ visible light communication [8-10]. All of those provide convenience for the realization of vehicular VLC without adding extra hardware.

The usual application model of vehicular VLC is presented in Figure 1. It can be applied mainly in the following ways:

(i) Vehicle to infrastructure (V2I) communication: the LEDs of the headlight and taillight are used as transmitters. PDs or image sensors on infrastructure are used as receivers. 


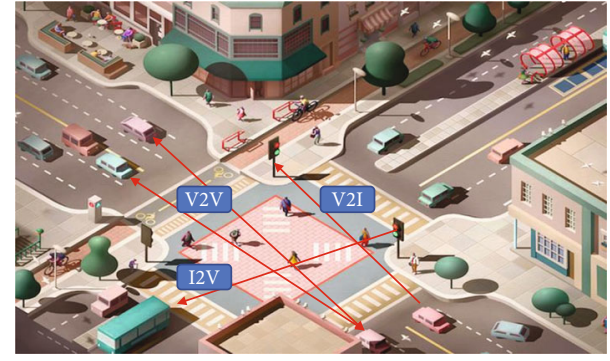

FIgURE 1: The usual application model of vehicular VLC.

(ii) Infrastructure to vehicle (I2V) communication: the LEDs of streetlights or traffic lights are used as transmitters. PDs or image sensors on cars are used as the receiver.

(iii) Vehicle to vehicle (V2V) communication: the LEDs of the headlight or taillight of the vehicle are used as the transmitters, and PDs or image sensors in the front or tail of vehicles are used as the receiver which can realize the communication between the front and rear vehicles.

This paper mainly studies the V2V VLC. The size of LEDs of headlight and taillight is limited that conforms to the requirements of vehicle configuration. When the LEDs of the headlight and taillight are used as the transmitters of VLC, the LED spacing under the premise of illumination makes it seem crowded in the long distance. Because of the diffuse reflection characteristics of LED, finite pixel size of the image sensor, and the small LED spacing, the blooming effect is caused. When there are many On LEDs around the Off LEDs, it is easy to be misidentified and reduced the reliability of the communication system. For the receiver terminal, most of the current studies focus on PDs or image sensors. The response speed of PD is fast, but the antiinterference ability is poor. Especially with the wide field of view, the direct radiation of background light (such as sunlight) will cause the received power much higher than the optical power of the transmitted signal. Even with using the optical filter, the expected performance is still not achieved. In contrast, the image sensor has a better spatial separation ability. Even under the influence of background light, it can still extract only the pixel information related to the transmitter and discard the noise information. So, the image sensor is selected as the receiver in this paper.

The taillights of the vehicle are always composed of multiple LEDs. In VLC, information can be transmitted by LED brightness, so that the taillights of the vehicle can transmit multibit information. At the receiving terminal, the area occupied by the taillight can be extracted based on image sensors; then, the statuses On and Off of LEDs in each frame can be identified through threshold comparison that fed to the decoder to retrieve the original data.

The influence of the blooming effect mainly depends on the size and spacing of the LED, and the adjacent LED interference is the major source of the blooming effect [11]. To ensure safety, V2V-VLC or I2V-VLC needs to identify the information sent by the transmitter quickly, which requires that the calculated amount of the receiver should not be too large and the threshold setting should be reasonable. To reduce the blooming effect, some researchers have done some studies on constant and dynamic threshold selection. A simple mathematical expression of interpixel interference (IPI) based on the assumption was proposed in reference [12]. When the distance was the same, the LED diffusion was the same and only interfered with the surrounding LEDs. The diffusion factor was estimated by the minimum mean square error (MMSE) criterion filter which can reduce IPI. The threshold was zero when decoding. But the blooming coefficient depends on the environmental state and varies with the environment $[13,14]$. A demodulation method for blurred images based on IS-VLC was proposed in reference [15]. The Gaussian filter was used to approximate the blooming effect, and the convolution formula of the optical power of the transmitted signal, blooming effect, and received signal was given. The threshold was selected (0.5) when decoding. Although this method has a small amount of calculation, it will make it impossible to solve owing to an irreversible blooming effect matrix. Compared with the fixed threshold, the dynamic threshold can be adjusted according to the state of the vehicle, which conforms to the actual situation. Some scholars have studied the dynamic threshold using the mean value method. Convolutional coding was used to reduce the number of logic level 1 in the transmission sequence so that the status On of LEDs in the image was less and solve the problem of intersymbol interference and reduce the blooming effect. In the actual communication, the probability that the status Off of LED is close to the status On of LED is small. If this average value was used as the threshold, it was easy to produce error [16]. A multifeature LED bit detection algorithm was proposed in reference [17] that using three new LED features, average greyscale ratio (AGR), gradient radial inwardness (GRI), and neighbour greyscale ratio (NGR). Then, Fisher linear discriminant analysis (FLDA) was applied combining the three features and the threshold was the mean of the projections of all LED feature vectors, which showed good robustness, but required a lot of computation and time. In addition, the dynamic threshold selection of the nonmean value method was also concerned. Second-order polynomial fitting was used to reduce the blooming effect of the CMOS sensor, histogram equalization, and Sobel filter to reduce the influence of background noise and improve the transmission distance. Finally, the threshold was determined by the thirdorder polynomial fitting of the original grayscale and the filtered grayscale, which improved the bit error rate, but this method was suitable for short-distance transmission [18, 19]. The intersymbol interference (ISI) caused by stray light in IS-VLC was analysed in reference [20] which calculated the expected value of SISI using permutation and combination theory and gave the calculation method of the threshold. According to the analysis, with the increase of distance, BER deteriorates faster, which was not conducive to long-distance transmission.

Different from the fixed threshold selection method, a novel dynamic threshold selection algorithm based on the 
idea of threshold selection of OOK is proposed in this paper. The Gaussian filter is used to approximate the blooming effect, and the interferences of surrounding LEDs are analysed. After this, the values of all-possible receiving power with different statuses of LEDs are found and two kinds of receiving optical power probability distribution curves at the statuses On and Off of LEDs are given. If two curves do not intersect, the mean of the minimum receiving power of the status On of LED and the maximum receiving power of the status Off of LED is selected as the threshold. Otherwise, the threshold can be found with the minimum BER using the probabilistic statistical method. This algorithm solves the problems of unreasonable value and large computation of the mean value method. Compared with the nonmean value method, the dynamic threshold selection algorithm of the proposed algorithm is more suitable for long-distance transmission. The simulation results show that the bit error rate has been better improved.

The structure of this paper is as follows: the second section introduces the V2V-VLC system, the third section introduces the calculation process of the threshold, the fourth section is the simulation and result analysis, and the fifth section is the summary of this paper.

\section{Fundamental of V2V-VLC System}

The architecture of the V2V-VLC system based on image sensors is illustrated in Figure 2. At the transmitting terminal, the transmitting data is encoded and modulated firstly, and logic levels 1 and 0 control the statuses On and Off of LEDs, respectively, in the LED array panel of the taillight. The information is transmitted by high-speed flashing LEDs and through the channel to the receiving terminal. Then, the image sensors capture the image of the LED array panel. Through digital image processing technology, the receiving power of each LED is compared with the threshold value which realizes decoding and recovers the original data. If it is greater than the threshold value, it is judged to be logic level 1. Otherwise, it is judged to be logic level 0 .

2.1. Transmitter. At the transmitter terminal, $m \times n$ highspeed flashing LEDs transmit information without causing harm to human eyes. Let the optical power of the LED array be $P(t)=\{P 1(t), P 2(t), \cdots, P i(t) \cdots, P m \times n(t)\} T(1 \leq i \leq m$ $\times n)$, the peak optical power of LED be $P_{\mathrm{LED}}$, and $P_{u, v, k}(k$ $=1,2, \cdots)$ be the coefficient determining the intensity of LED with row $u$ and column $v$ which will become logic levels 0 and 1 after optical OOK modulation [21]. Then, $P_{u, v}(t)$ can be expressed as follows:

$$
P_{u, v}(t)=\sum_{k} P_{u, v, k} \cdot P_{\mathrm{LED}} \cdot f\left(t-(k-1) T_{b}\right) .
$$

$f(t)$ is a rectangular pulse function that can be expressed as follows:

$$
f(t)= \begin{cases}1, & 0 \leq t<T_{b}, \\ 0, & \text { otherwise }\end{cases}
$$

2.2. Channel Gain. Due to the large receiving field angle of the image sensor, line of sight (LOS) can be easily realized. In this paper, we only focus on the LOS channel when we analyse the channel characteristics.

The LED-emitting light source can be modeled by the Lambert radiation model, and its radiation intensity can be expressed as follows [22]:

$$
R_{0}(\phi)=\frac{(m+1)}{2 \pi} \cdot P_{\mathrm{LED}} \cdot \cos ^{m}(t), \quad \phi \in\left[-\frac{\pi}{2}, \frac{\pi}{2}\right],
$$

where $\phi$ is the radiation angle of the LED, $m$ is the Lambert radiation number, and its value is related to the power intensity angle of the light source plate, which is expressed as follows:

$$
m=-\frac{\ln 2}{\ln \cos \Phi_{1 / 2}}
$$

where $\Phi_{1 / 2}$ is the half-power intensity angle of LED.

In the absence of Gaussian blurring and noise interference, the DC gain of the LOS channel can be expressed as follows [23]:

$$
H_{t}(0)=\frac{A_{p}}{u^{2}} \bullet R_{0}(\phi) \cdot \cos (\varphi)
$$

where $\varphi$ is the incident angle of LED, $u$ is the distance between transmitter and receiver, and $A_{p}$ is the incident pupil area of IS lens.

The received optical power of the $i$-th LED without interference can be expressed as follows:

$$
y_{o, i}(t)=c \bullet H_{t}(0) \bullet P_{i}(t)
$$

where $c$ is the coefficient of light-to-current transfer efficiency.

2.3. Channel Noise. Gaussian blurring is caused by the diffuse reflection of LEDs and size limitation of the image sensor. After channel transmission, the LED on the image sensor no longer focuses on a point but diffuses into a circle, which is called Airy disk [24], and its point spread function (PSF) can be approximated as Gaussian function. The expression is as follows:

$$
g(p, q)=\frac{1}{2 \pi \sigma^{2}} \cdot \exp \left(-\frac{p^{2}+q^{2}}{2 \sigma^{2}}\right)
$$

where $\sigma^{2}$ is the variance of Gaussian function and $p$ and $q$ are the horizontal and vertical components of the distance to the original point.

The point spread of LED affects the reception of adjacent LED, which is called spatial intersymbol interference (SISI), and can be modeled as the convolution of the input signal and Gaussian blurring function. The interference of the 


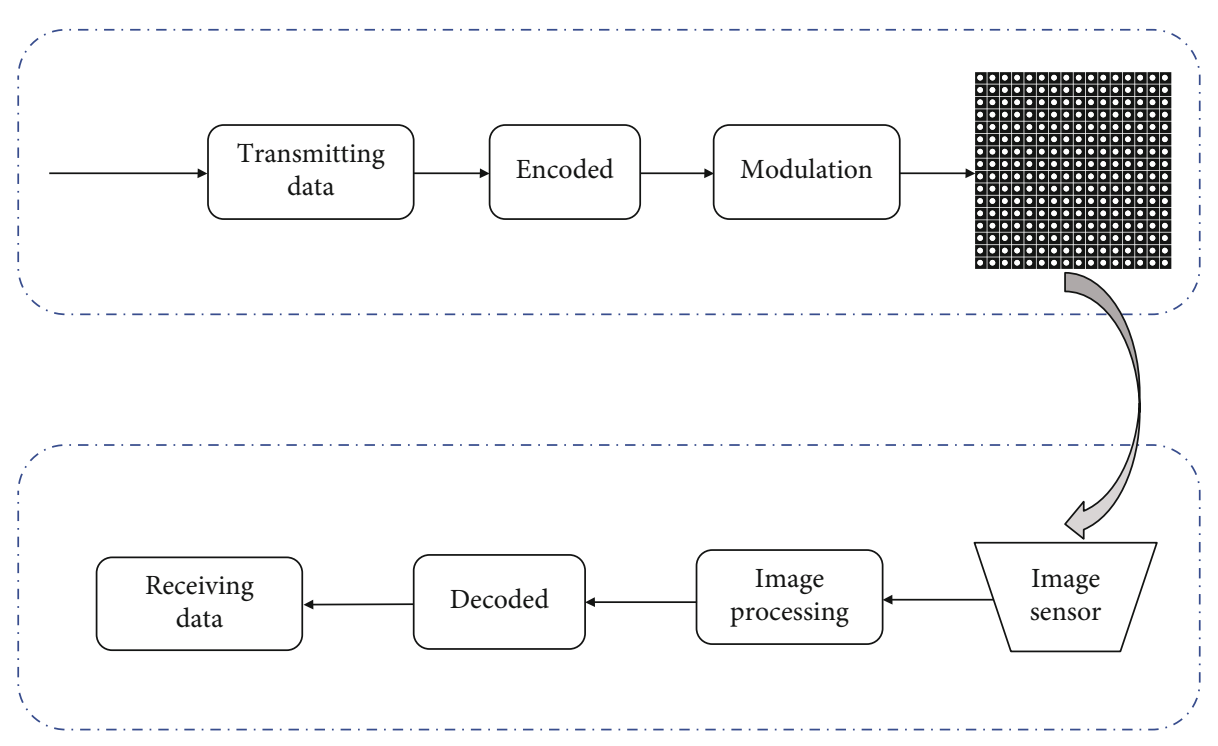

FIgURE 2: The architecture of the V2V-VLC system based on image sensors.

$j$-th LED to the $i$-th LED can be expressed as follows:

$$
S_{i, j}=\iint P\left(x_{j}, y_{j}\right) \cdot g\left(r_{i, j}^{\prime}\right) d x d y
$$

where $P\left(x_{j}, y_{j}\right)=c \bullet H_{t}(0) \bullet P_{j}(t)$.

In the LED array, if the interval between the LEDs is $d$, the distance between the $j$-th LED and the $i$-th LED can be expressed as follows:

$$
r_{i, j}=d \sqrt{\left(x_{i}-x_{j}\right)^{2}+\left(y_{i}-y_{j}\right)^{2}} .
$$

According to the imaging principle of the image sensor, when the object distance is far greater than the image distance, the spacing between the $j$-th LED and the $i$-th LED on the image sensor can be expressed as follows:

$$
r_{i, j}^{\prime}=\frac{r_{i, j} \bullet \mathrm{FL}}{u},
$$

where FL is the focal length and $u$ is the distance between the transmitter and the receiver.

From the formula of spatial intersymbol interference, with the increase of $\sigma$, the interference between symbols increase, and vice versa. It is called the blooming coefficient or blooming factor. With the increase of the interval of surrounding LEDs, the interference between the symbols becomes small. When the two LEDs are far away, the interference between them is almost zero that is the same as the effect of our actual human eye observation. To reduce the SISI between the LEDs, it is necessary to choose the appropriate value of $\sigma$ and LEDs' interval.

Besides SISI, ambient light noise also exists. This noise power can be calculated through ambient light power multiplied by the reflection coefficient of the LED surface.
2.4. Receiver. The optical power received at the receiving end can be expressed as follows:

$$
Y=c H \times P+N
$$

where $c$ is the coefficient of light-to-current transfer efficiency, $H$ is the channel characteristic matrix, $P$ is the input optical signal, and $N$ is the channel noise.

According to the channel characteristic analysis in the previous section, the channel characteristic matrix can be expressed as follows:

$$
H=H(0)+G \text {. }
$$

$H(0)$ is the DC gain matrix of the channel, which has value only on the diagonal. $G$ is the SISI matrix.

Let the received optical power be $Y(t)=\left\{y_{1}(t), y_{2}(t)\right.$, $\left.\cdots, y_{i}(t), \cdots, y_{m \times n}(t)\right\}^{T}(1 \leq i \leq m \times n)$. It can be expressed as follows:

$$
y_{i}(t)=c\left(H_{t}(0) \cdot P_{i}(t)+\sum_{j=1, j \neq i}^{m \times n} S_{i, j}+n_{i}\right) .
$$

Compared with the threshold value, if the received optical power is greater than the threshold value, it is judged to be logic level 1, and if it is less than the threshold value, it is judged to be logic level 0 . Then, the original data can be retrieved.

\section{Threshold Scheme}

The rationality of the threshold is related to the performance of BER. The distance between the transmitter and the receiver of $\mathrm{V} 2 \mathrm{~V}$-VLC is constantly changing due to the continuous movement of vehicles. As the channel characteristic matrix changes with distance, the received signals are also different which makes the threshold setting challenging. 
In Reference [16], the threshold is an average brightness. Before transmitting information, three groups of Barker sequence with the length of 13 are inserted. In the coding method, +1 represents that the LED lights in this frame are all On, and -1 represents that the LED lights are all off. There are 39 guide frames in total, of which 27 are all On. By calculating the total brightness of these 27 images and then dividing by the number of LEDs, an average value is obtained. The average value is used as the threshold value to judge whether the LED on the receiver is On or Off. The disadvantage of this method is that when the LEDs on the panel are all On, the mutual interference will increase, and the average value will also increase. In the actual communication, the probability that the off LED is close to the full light of the LED is small. If this average value is used as the threshold, it is easy to produce misjudgement. In order to make the comparison more intuitive, the average value of the LED's minimum received power with the overall status On and the LED's maximum received power with the overall status Off is selected as the threshold value that the parameters are consistent with the proposed method in this paper.

In Reference [20], the expected value of SISI is derived by permutation and combination theory, and the optimized threshold is calculated according to the selection strategy of judgment threshold in OOK; hereinafter, this method is referred to as the expected threshold method. This threshold is half of the sum of the received value of LOS channel, the average value of SISI, and the noise of LED optical power, which reflects the role of SISI in threshold judgment. In the actual communication, LED is mainly affected by the surrounding LED. Therefore, this paper only selects the SISI expected value of the surrounding LED and then obtains the threshold according to the method in reference [20], to maintain the consistency of parameter selection and facilitate simulation comparison.

SISI is mainly influenced by surrounding LEDs, and its schematic diagram is shown in Figure 3. $\mathrm{LED}_{R, C}$ is affected by the surrounding eight LEDs; their illuminance is related to the total interference value.

In VLC OOK modulation, the probability of the statuses On and Off of LEDs is equal to $1 / 2$, so there are two statuses of $\operatorname{LED}_{R, C}$ and a total of $2^{8}=256$ statuses On and Off of the eight neighbour LEDs. Therefore, according to formulas (7) and (8), we can calculate the influence of 256 different lighting conditions on the surrounding eight LEDs and add the received value of $\mathrm{LED}_{R, C}$ itself to get the optical power value under the statuses On and Off of LEDs. Through the probability statistics of the optical power value, the distribution of different statuses is obtained.

There are two kinds of receiving optical power probability distribution curves at the statuses On and Off of LEDs.

(i) The receiving optical power probability distribution curves do not intersect at two different statuses. The minimum receiving power of the status On of LED is greater than the maximum receiving power of the status Off of LED, that is $Y_{\mathrm{ON}, \min }>Y_{\mathrm{OFF}, \max }$.

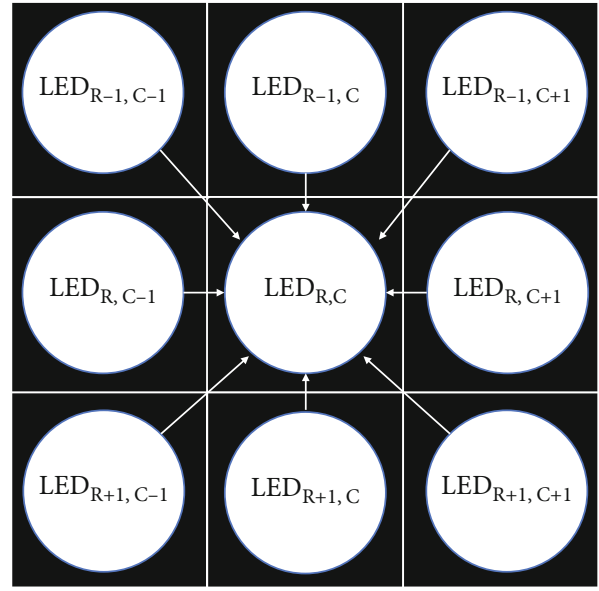

FIgURE 3: Schematic diagram of SISI.

Whether the status is On or Off of LED, the received optical power is quite different. The mean value of $Y_{\mathrm{ON} \text {,min }}$ and $Y_{\mathrm{OFF} \text {,max }}$ can be selected as the threshold to realize an error-free decision

(ii) The receiving optical power probability distribution curves intersect at two different statuses. The minimum receiving power of the status On of LED is less than the maximum receiving power of the status Off of LED, that is, $Y_{\mathrm{ON} \text {,min }} \leq Y_{\mathrm{OFF} \text {,max }}$. There will exist misjudgement, and the selection of threshold will affect the BER. In the traditional OOK modulation, the sampling value of the receiver is a Gaussian random variable, and its one-dimensional probability density function can be obtained. When the partial derivative is equal to 0 , the best threshold can be obtained. By the statuses of the surrounding LEDs, the theoretical value of the received power is discrete and so is the corresponding probability distribution. It is very difficult to fit the curve. Therefore, the traditional OOK threshold selection method is difficult to achieve for V2V VLC. In this paper, the selection method is used to determine the threshold. For $Y_{\mathrm{ON} \text {,min }} \leq Y_{\mathrm{OFF} \text {,max }}$, the initial threshold is $\mathrm{Th}_{u}=$ $Y_{\mathrm{ON} \text {,min }}$. Calculating the probability of $Y_{\mathrm{ON}}<\mathrm{Th}_{u}$ and $Y_{\mathrm{OFF}>} \mathrm{Th}_{u}$ under this threshold, the sum of probability corresponding received power is the BER. Then, the threshold is iterated to the next received optical power value, and the BER is calculated until the iteration goes to $Y_{\mathrm{OFF} \text { max }}$. After the iteration, the received optical power with the lowest BER is selected as the threshold $\mathrm{Th}_{u}$

The proposed algorithm is as follows.

According to this algorithm, the probability distribution curves of LED optical power at different communication distances are calculated, as shown in Figure 4.

As can be seen from Figure 4, the intersection of receiving optical power at different distances is also different. When the distance is close, such that the communication distance is 30 meters, the two optical power distributions 


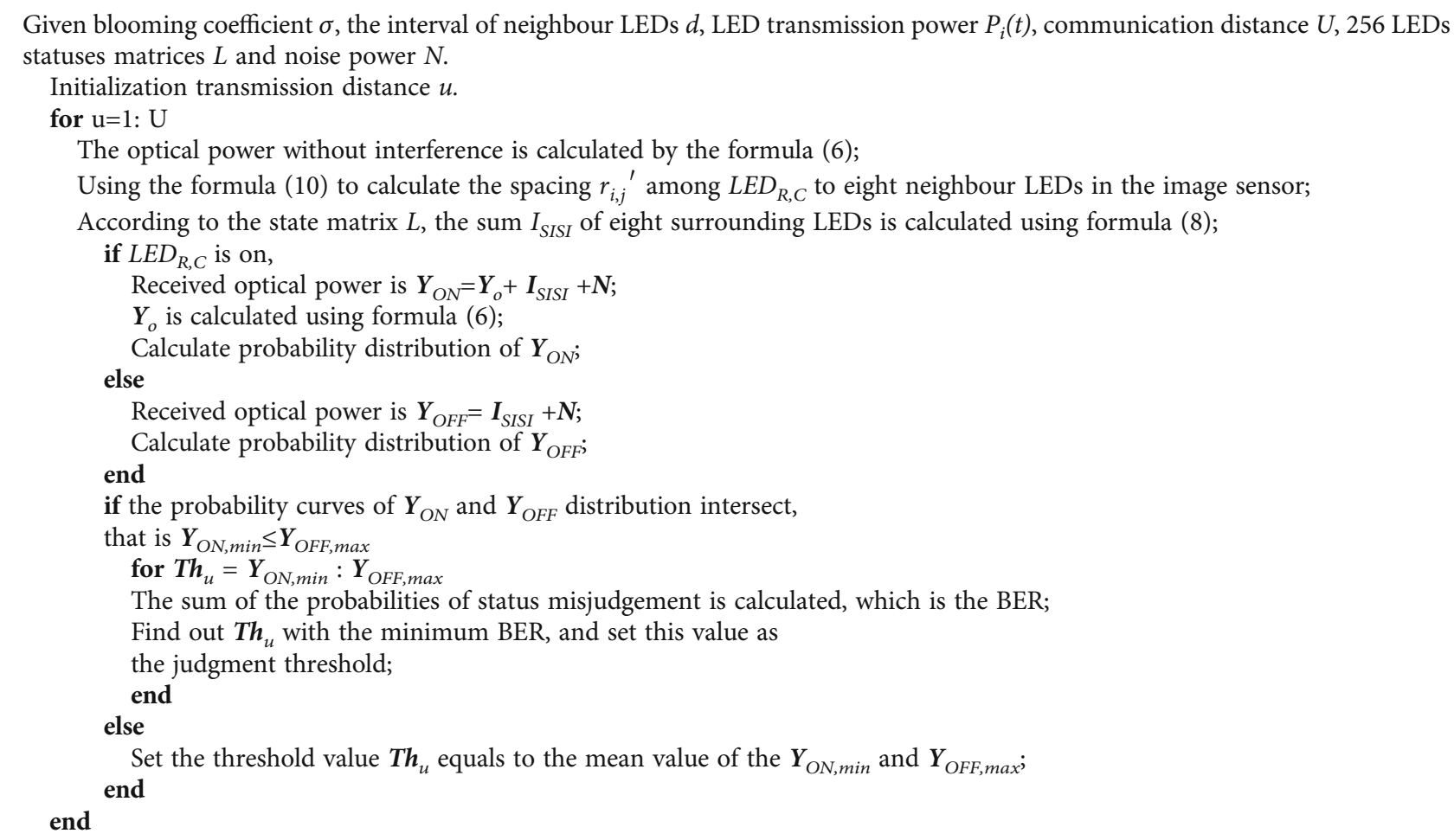

Algorithm 1: Threshold calculation algorithm based on probability distribution.

do not intersect, which shows that the receiving optical power at the statuses On and Off of LEDs is quite different. It also shows that the influence of surrounding LEDs is small, and the receiving optical power of LEDs at the status Off will not be increased. When the distance is longer, such as from $50 \mathrm{~m}$ to $70 \mathrm{~m}$, with the increasing distance, more and more parts of the receiving optical power distributions coincide at different statuses. This is because of the increase of the distance, the interval between the LEDs in the image sensor is getting smaller and smaller, and the surrounding LEDs are getting closer and closer, which makes the influence of the adjacent LEDs more and more serious. If the threshold selection is not reasonable, the status Off of LED will be identified as On, and the status On of LED may be identified as Off, resulting in the deterioration of BER.

\section{Results and Discussion}

4.1. Simulation Environment. In this paper, computer simulation is used to verify the effectiveness of the proposed algorithm. The core parameter settings are shown in Table 1 . The size of the LED array is $16 \times 16$ with a total of 256 LEDs. The radius of LEDs is $3 \mathrm{~mm}$, the interval is $15 \mathrm{~mm}$, the halfpower angle is 60 degrees, and the LEDs' optical power is $0.2 \mathrm{~W}$, which is the common LED-rated power of automobile taillight. The exposure time of the image sensor at the receiving end is the same as the flashing period of the LED, its focal length is $35 \mathrm{~mm}$, the receiving field angle is 70 degrees, and the pixel size is $2.2 \mu \mathrm{m}$, which is the pixel size that can be achieved at present. The communication dis- tance is $20-70$ meters, which is far greater than the image distance. The modulation mode is optical OOK modulation, and the Gaussian blooming coefficient is 3. To verify the effectiveness of the algorithm, different LED intervals, Gaussian blooming coefficient, and communication distance are used for simulation, and the BER of the expected threshold method, the average threshold method, and the threshold proposed in this paper are compared.

\subsection{Simulation Results and Analysis}

4.2.1. BER Performance with Different LED Intervals. Figure 5 shows the BER performance with different LED intervals when the communication distance is $60 \mathrm{~m}$ and the Gaussian ambiguity coefficient is 3, where the LED interval is $5-25 \mathrm{~mm}$ and the comparison is made every $5 \mathrm{~mm}$.

As can be seen from Figure 5, with the increase of LED interval, the BER performance of the three methods improves continuously. According to Equations (7)-(10), the LED spacing on the image sensor is also increasing with the increasing of the actual LED interval, causing the interferences of the $j$-th LED to the $i$-th LED to decrease which makes the receiving optical power difference between the statuses On and Off larger. When the LED interval is large enough (over $25 \mathrm{~mm}$ ), the bit error rate is almost zero. Regarding the comparison between the proposed scheme and the existing scheme, the threshold of the proposed scheme is calculated based on the probability distribution of receiving powers with the minimum BER. However, when $Y_{\mathrm{OFF} \text { max }}$ is larger than $Y_{\mathrm{ON} \text {,min }}$, the threshold of the average 


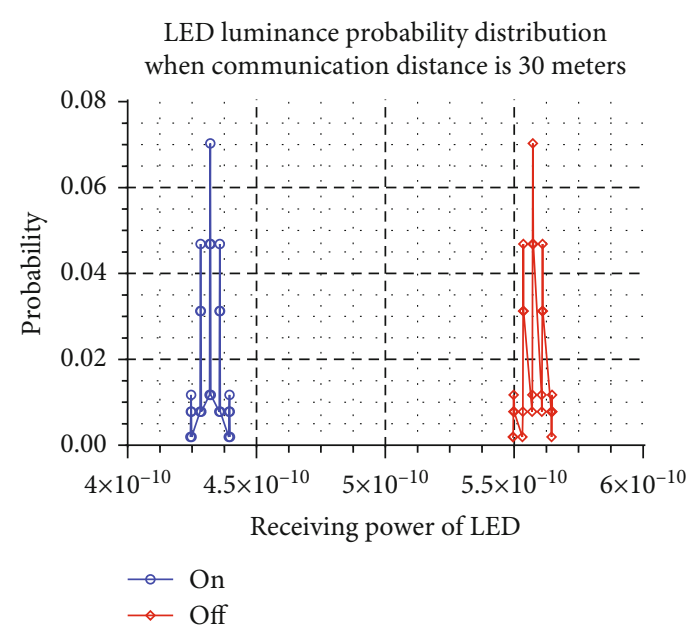

(a)

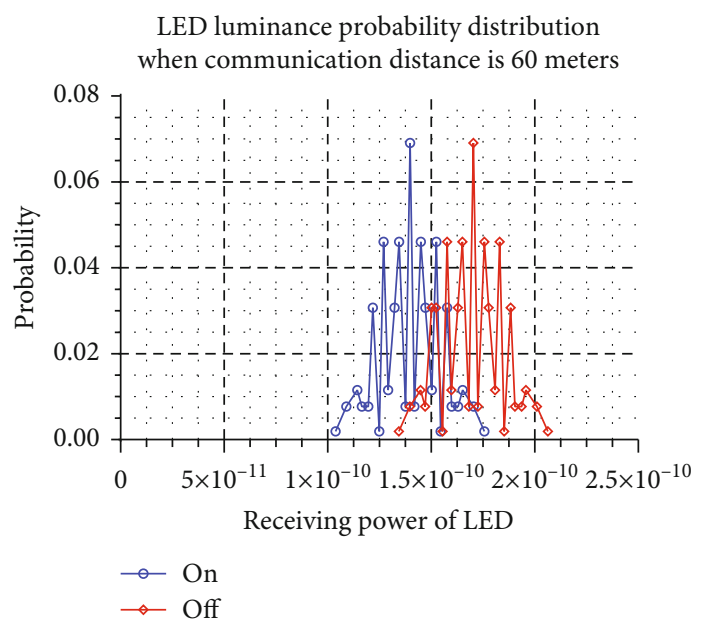

(c)

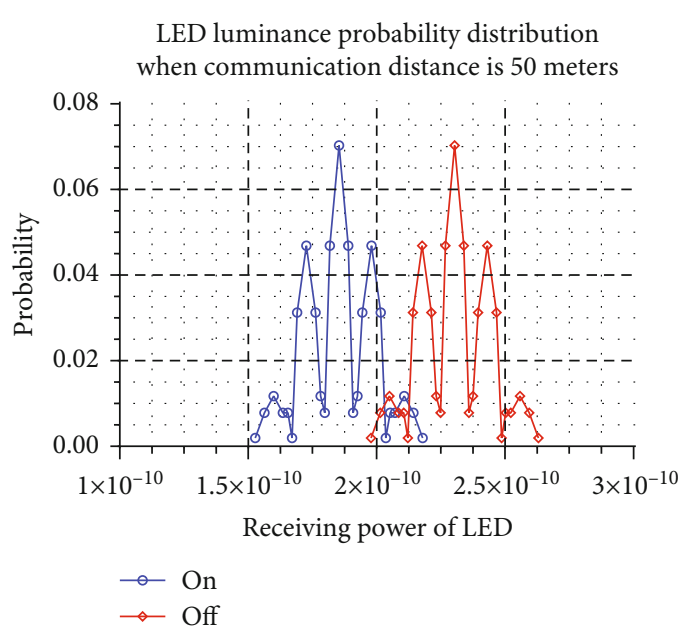

(b)

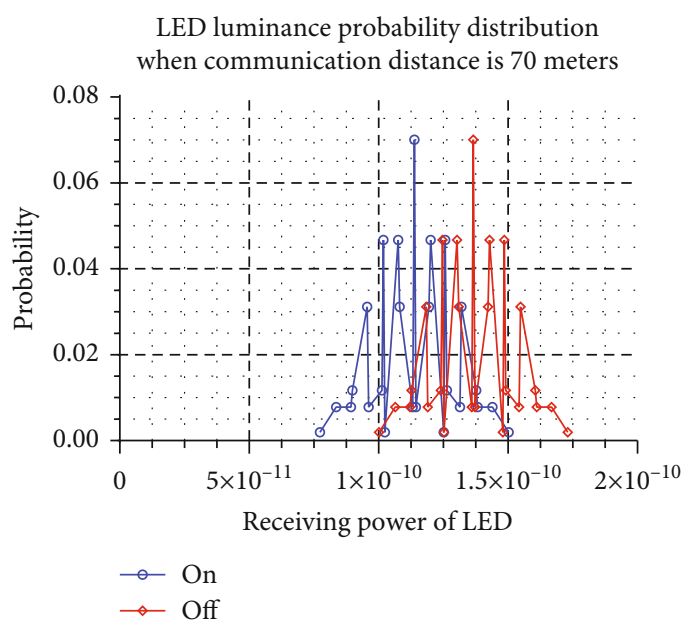

(d)

FIGURE 4: The receiving optical power distribution curves with different communication distances. (a) The communication distance is 30 m; (b) the communication distance is $50 \mathrm{~m}$; (b) the communication distance is $60 \mathrm{~m}$; (b) the communication distance is $70 \mathrm{~m}$.

TABLE 1: Core parameter setting for simulation.

\begin{tabular}{lc}
\hline LED array & $16 \times 16$ \\
\hline Led radius & $3 \mathrm{~mm}$ \\
Led spacing & $18 \mathrm{~mm}$ \\
Half power angle & 60 degrees \\
Rated power & $0.2 \mathrm{~W}$ \\
Focal length & $35 \mathrm{~mm}$ \\
Receiving field angle & 70 degrees \\
Pixel size & $2.2 \mu \mathrm{m} \times 2.2 \mu \mathrm{m}$ \\
Gaussian blooming coefficient & 3 \\
Communication distance & $20-70 \mathrm{~m}$ \\
Modulation mode & OOK \\
\hline
\end{tabular}

value method is the mean value of $Y_{\mathrm{ON} \text {,min }}$ and $Y_{\mathrm{OFF} \text {,max }}$, and it is easier to misjudge. Also, the threshold of the expected threshold method is related to the expected value of SISI which is a large difference. It can be seen from the comparison that the proposed threshold scheme is superior to the other two methods; especially when the interval is $15 \mathrm{~mm}$ or $20 \mathrm{~mm}$, the BER improvement speed is significantly faster than the expected threshold method and the average value method.

Although the performance of BER is improved obviously with the increasing of LED spacing, it cannot increase without limit, which is not in line with the actual application of vehicles. Because of the size limitation of the car taillight panel, the larger LED spacing will reduce the number of LEDs. In order to meet the needs of lighting, we need to increase the power of LED, and the increasing power of LED will also increase the interference of adjacent LED. Therefore, it is necessary to jointly optimize the LED spacing and number in future research to meet the requirements of lighting and better communication simultaneously.

4.2.2. BER Performance with Different Blooming Coefficients. Figure 6 shows the BER performance of different LED blooming coefficients when the communication distance is $60 \mathrm{~m}$ and the LED spacing is $18 \mathrm{~mm}$. The blooming 


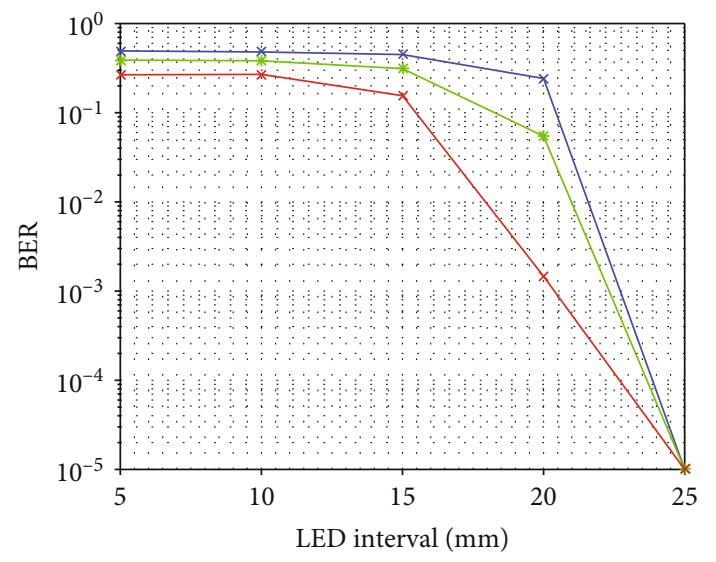

$$
\begin{aligned}
& * \text { Expectation threshold method } \\
& * \text { Average threshold method } \\
& * \text { Proposed method }
\end{aligned}
$$

FIGURE 5: BER performance with different LED intervals.

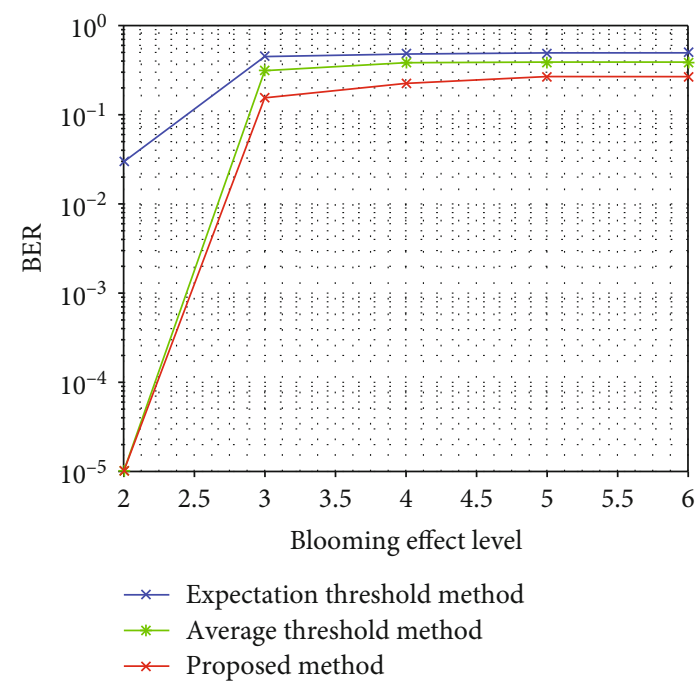

Figure 6: BER performance with different blooming coefficients.

coefficient is $2-6$, and the BER performance is compared every time the blooming coefficient increases by 1 .

It can be seen from Figure 6 that the BER performance deteriorates with the increase of the blooming coefficients. According to Equations (7) and (8), with the increase of the blooming coefficient, the interferences of the $j$-th LED to the $i$-th LED increase which makes the receiving optical power difference between the statuses On and Off smaller. While the blooming coefficient increases, there are more intersecting points between the two curves. Especially for the status Off of LEDs, if there are several On LEDs nearby, the received power will be increased which makes $Y_{\mathrm{OFF} \text { max }}$ larger than $Y_{\mathrm{ON} \text {,min }}$. In this simulation range, the threshold strategy proposed in this paper is superior to the other two methods. When the blooming coefficient is less than 3 , the improvement effect is more obvious. When the blooming coefficient is 3 , the proposed method is $65.5 \%$ and $50.4 \%$

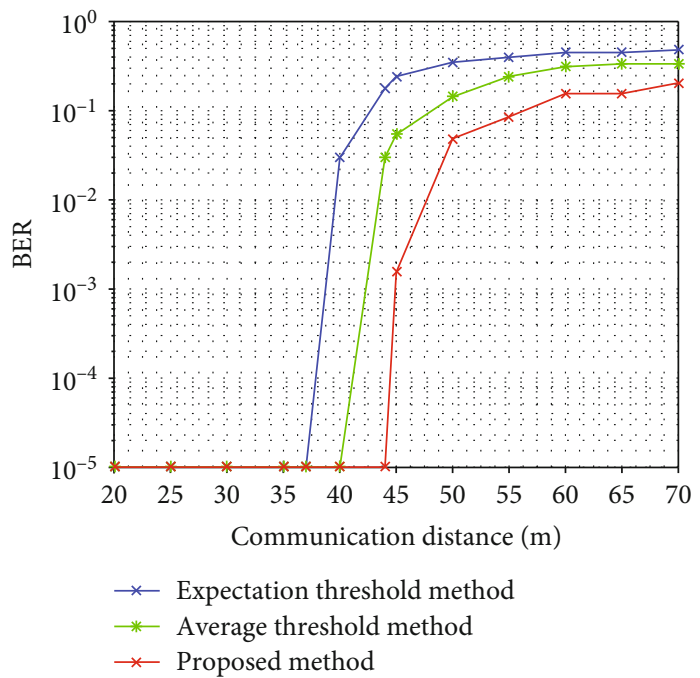

FIGURE 7: BER performance with different communication distances.

better than the expected threshold method and the average value method, respectively, which confirms that the proposed method has strong ability with the blooming coefficients changing.

The restoration of blurring images is one of the current research hotspots. Different from ordinary image processing, in V2V VLC based on image sensors, it needs to recover and detect the received image quickly, which needs a more accurate algorithm to achieve. The noise in the channel is mainly Gaussian blur and ambient light noise. Gaussian blurring is a kind of multiplicative noise, and the ambient light noise is additive noise. When the combination of the two affects the communication performance, additive noise can be considered to approximate multiplicative noise, so that the noise in the channel is all additive noise, and the receiver can filter it easily. In addition, blank pixels can be artificially inserted into the received image, and the Gaussian mixture model is used to fit the received signal to improve the influence of Gaussian blurring.

\subsubsection{BER Performance with Different Communication} Distances. Figure 7 shows the BER performance with different communication distances when the LED spacing is $18 \mathrm{~mm}$ and the Gaussian ambiguity coefficient is 3 , where the communication distance is $20-70 \mathrm{~m}$ and the comparison is made every $5 \mathrm{~m}$.

As can be seen from Figure 7, the BER performance deteriorates with the increase of communication distance. According to Equations (7)-(10), with the increasing of communication distance, the area occupied by the whole LED array on the image sensor is getting smaller, the spacing between the LEDs is getting closer, and the surrounding LED interference is getting bigger and bigger. Most times, we can only recognize the area of the LED array panel on the image sensor and divide the area equally in that each block represents an LED. It is possible that $Y_{\mathrm{OFF} \text { max }}$ is larger than $Y_{\mathrm{ON} \text {,min }}$. In this simulation range, the proposed threshold strategy is better than the other two methods and can 
achieve no errors before $44 \mathrm{~m}$. With the increase of distance, the BER improvement effect is more and more obvious. At $70 \mathrm{~m}$, the BER of the proposed scheme is improved by $39 \%$ and $57 \%$ compared with the average threshold method and the expected threshold method, respectively, which confirms to be more suitable for long-distance communication.

Although the BER performance is better when the communication distance is close, in order to meet the needs of safety, vehicles must keep a certain distance from each other. Especially when driven on the highway, vehicles must be kept at a safe distance to avoid accidents. In future research, we can reduce the number of On LEDs in the LED array using pre-coding (such as convolutional coding), especially the number of On LEDs around the Off LEDs, to reduce the interference between LEDs. However, the reduction of the number of bright LEDs will cause a lack of lighting function. Under the condition of meeting the lighting conditions, the appropriate layout optimization of the status On or Off LEDs needs more in-depth research. In addition, multiple LED light groups can transmit 1-bit information, which reduces the transmission rate, but improves the BER.

\section{Conclusions}

SISI is an important factor affecting the bit error rate of V2V VLC, which mainly depends on the parameters such as LED spacing, ambiguity coefficient, and communication distance. With different parameters, the influence of SISI is also different. The smaller the LED interval, the larger the blooming coefficient or the longer the communication distance will cause the increase of SISI and then affect the final decision.

This paper analyses the communication framework of V2V VLC based on image sensors, calculates the possible SISI caused by surrounding LEDs, and proposes a threshold selection strategy, which is verified by simulation. By changing the influence factors of SISI such as LED spacing, blooming coefficient, and communication distance, the BER performances of the proposed threshold method, the expected threshold method, and the average threshold method are compared. The proposed method is superior to the other two methods; especially, it can be error-free before $44 \mathrm{~m}$, and the BER is improved significantly. In a future work, we can use mathematical methods to fit the probability distribution curve of the received power and calculate the optimal solution. We can also explore new coding methods to reasonably configure the ratio of the statuses On and Off of LEDs in each frame or optical filtering methods to reduce interference and further improve BER.

\section{Data Availability}

The data used to support the findings of this study are available from the corresponding author upon request.

\section{Conflicts of Interest}

The authors declare that there is no conflict of interest regarding the publication of this paper.

\section{Acknowledgments}

This research was funded by the National Natural Science Foundation of China (grant number 61961007); the Basic Ability Improvement Project of Young and Middle-aged Teachers in Guangxi Universities (grant number 2021KY0217); the Dean Project of the Guangxi Key Laboratory of Wireless Wideband Communication; the Dean Project of Ministry of Education Key Lab. of Cognitive Radio and Information Processing (grant number CRKL170110); and the School of Information and Communication, Guilin University of Electronic Technology, Guilin, China.

\section{References}

[1] J. J. Shea, "Book reviews [6 books reviewed]," IEEE Electrical Insulation Magazine, vol. 37, no. 3, pp. 45-47, 2021.

[2] P. He, Y. X. Shi, T. Meng et al., "Recent advances in white light-emitting diodes of carbon quantum dots," Nanoscale, vol. 12, no. 8, pp. 4826-4832, 2020.

[3] M. Usman, S. Malik, and M. Munsif, "AlGaN-based ultraviolet light-emitting diodes: challenges and opportunities," Luminescence, vol. 36, no. 2, pp. 294-305, 2021.

[4] R. Z. Liu and K. Xu, "Blue perovskite light-emitting diodes (LEDs): a minireview," Instrumentation Science \& Technology, vol. 48, no. 6, pp. 616-636, 2020.

[5] S. Arai, M. Kinoshita, and T. Yamazato, "Optical wireless communication: a candidate $6 \mathrm{G}$ technology?," IEICE Transactions on Fundamentals of Electronics, Communications and Computer Sciences, vol. E104.A, no. 1, pp. 227-234, 2021.

[6] S. Ariyanti and M. Suryanegara, "Visible light communication (VLC) for 6G technology: the potency and research challenges," in 2020 Fourth World Conference on Smart Trends in Systems, Security and Sustainability (WorldS4), pp. 490493, London, UK, 2020.

[7] N. Chi, Y. Zhou, Y. Wei, and F. Hu, "Visible light communication in 6G: advances, challenges, and prospects," IEEE Vehicular Technology Magazine, vol. 15, no. 4, pp. 93-102, 2020.

[8] A. Memedi and F. Dressler, "Vehicular visible light communications: a survey," IEEE Communications Surveys and Tutorials, vol. 23, no. 1, pp. 161-181, 2021.

[9] E. Eso, Z. Ghassemlooy, S. Zvanovec, J. Sathian, M. M. Abadi, and O. I. Younus, "Performance of vehicular visible light communications under the effects of atmospheric turbulence with aperture averaging," Sensors, vol. 21, no. 8, p. 2751, 2021.

[10] S. Caputo, L. Mucchi, F. Cataliotti, M. Seminara, T. Nawaz, and J. Catani, "Measurement-based VLC channel characterization for I2V communications in a real urban scenario," Vehicular Communications, vol. 28, p. 100305, 2021.

[11] T. H. Do and M. Yoo, "Multiple exposure coding for short and long dual transmission in vehicle optical camera communication," IEEE Access, vol. 7, pp. 35148-35161, 2019.

[12] T. Kasashima, T. Yamazato, H. Okada, T. Fujii, T. Yendo, and S. Arai, "Interpixel interference cancellation method for roadto-vehicle visible light communication," in 2013 IEEE 5th International Symposium on Wireless Vehicular Communications (WiVeC), pp. 1-5, Dresden, Germany, 2013.

[13] T. Yamazato, "Overview of visible light communications-with emphasis on image sensor communications,", in 2017 23rd Asia-Pacific Conference on Communications (APCC), pp. 16, Perth, WA, Australia, 2017. 
[14] M. Kinoshita, T. Yamazato, H. Okada et al., "Motion modeling of mobile transmitter for image sensor based I2V-VLC, V2IVLC, and V2V-VLC," in 2014 IEEE Globecom Workshops (GC Wkshps),, pp. 450-455, Austin, TX, USA, 2014.

[15] Y. Ohira, S. Arai, T. Yendo et al., "Novel demodulation scheme based on blurred images for image-sensor-based visible light communication," in 2015 IEEE Globecom Workshops (GC Wkshps), pp. 1-6, San Diego, CA, USA, 2015.

[16] D. Vuong and M. Yoo, "Interpixel interference mitigation in visible light communication using image sensor," IEEE Access, vol. 6, pp. 45543-45551, 2018.

[17] T. H. Do and M. Yoo, "A multi-feature LED bit detection algorithm in vehicular optical camera communication," IEEE Access, vol. 7, pp. 95797-95811, 2019.

[18] C. W. Chow, C. Y. Chen, and S. H. Chen, "Enhancement of signal performance in LED visible light communications using mobile phone camera," IEEE Photonics Journal, vol. 7, no. 5, pp. 1-7, 2015.

[19] C. W. Chow, C. Y. Chen, and S. H. Chen, "Visible light communication using mobile-phone camera with data rate higher than frame rate," Optics Express, vol. 23, no. 20, pp. 2608026085, 2015.

[20] P. Liu, P. Zheng, S. Yang, and Z. Chen, "Modeling and analysis of spatial inter-symbol interference for RGB image sensors based on visible light communication," Sensors, vol. 19, no. 22, 2019.

[21] S. Arai, S. Mase, T. Yamazato et al., "Experiment on hierarchical transmission scheme for visible light communication using LED traffic light and high-speed camera," in 2007 IEEE 66th Vehicular Technology Conference, pp. 2174-2178, Baltimore, MD, USA, 2007.

[22] M. Akanegawa, Y. Tanaka, and M. Nakagawa, "Basic study on traffic information system using LED traffic lights," IEEE Transactions on Intelligent Transportation Systems, vol. 2, no. 4, pp. 197-203, 2001.

[23] T. Yamazato, M. Kinoshita, S. Arai et al., "Vehicle motion and pixel illumination modeling for image sensor based visible light communication," IEEE Journal on Selected Areas in Communications, vol. 33, no. 9, pp. 1793-1805, 2015.

[24] B. Zhang, J. Zerubia, and J. C. Olivo-Marin, "A study of Gaussian approximations of fluorescence microscopy PSF models," in Proceedings of Conference on Three-Dimensional and Multidimensional Microscopy-Image Acquisition and Processing XIII, San Jose, CA, USA, 2006. 